\title{
Observation of Marfes in the Wendelstein 7-X stellarator with inboard limiters
}

\author{
U. Wenzel ${ }^{a}$, C. Biedermann ${ }^{a}$, G. Kocsis ${ }^{b}$, T. Szepesi ${ }^{b}$, B. D. \\ Blackwell $^{c}$, S. Klose ${ }^{a}$, J. Knauer ${ }^{a}$, M. Krychowiak ${ }^{a}$, L.

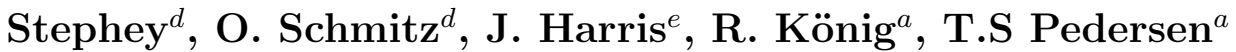 \\ and the W7-X team \\ ${ }^{a}$ Max-Planck-Institut für Plasmaphysik (IPP), EURATOM Association, \\ Wendelsteinstr. 1, 17491 Greifswald, Germany \\ ${ }^{b}$ Wigner RCP, RMI, Konkoly Thege 29-33, H-1121 Budapest, Hungary \\ ${ }^{c}$ Australian National University, Plasma Research Laboratory, Canberra, ACT, \\ Australia \\ ${ }^{d}$ University of Wisconsin, Madison, Wisconsin 53706, USA \\ ${ }^{e}$ Oak Ridge National Laboratory, Oak Ridge, Tennessee 37831, USA
}

\begin{abstract}
In the first operational campaign of the Wendelstein 7-X (W7-X) stellarator we observed Marfe-like radiation belts at the plasma edge. We describe and analyze the behaviour of these radiation belts, especially with respect to the high density inside the belt, with a set of suitable diagnostics. We present evidence suggesting that these Marfe-like radiation belts are triggered by a drop of the edge temperature below $30 \mathrm{eV}$. From this observation we draw the conclusion that the physical cause of Marfes in tokamaks and Marfe-like radiation belts in W7-X is the same, namely radiative condensation. Furthermore, both exhibit the device symmetry, i.e. axisymmetry in a tokamak and the fivefold stellarator symmetry in W7-X.
\end{abstract}




\section{Introduction}

$\mathrm{W} 7-\mathrm{X}$ is a large stellarator with a major radius $\mathrm{R}=5.5 \mathrm{~m}$ optimized for low neoclassical transport, a small bootstrap current and a good fast ion confinement [1]. For the first experimental campaign, five limiters were installed in the bean-shaped planes at the inboard side. W7-X was fuelled only by gas puffing. With limiters and no divertor, exhaust of neutrals was not effective due to the low neutral pressure in front of the pumping duct. Earlier in the campaign, owing to limited wall-conditioning, outgassing from the walls would be so intense that the plasmas would start shrinking even before making contact with the limiters. Later, discharges had prolonged contact with the limiters. Still, in some of these discharges, wall outgassing would set in and the plasma would shrink and collapse. In a number of those discharges, the beginning of the end of the plasma would feature a pronounced neutral pressure spike, and an initially poloidally localized, rotating radiation belt before the plasma would shrink and extinguish itself. Further details and an overview of the main results of the first campaign can be found in $[2]$.

Poloidally localized radiation belts are frequently observed in tokamaks and are known as multifaceted asymmetric radiation from the edge (Marfes). An early observation was reported from ALCATOR [3]. Subsequently, Marfes were found in many other tokamaks, including JET [4], ASDEX Upgrade [5], FTU [6] and Textor [7]. It is experimentally proven that a Marfe is a high density, low temperature plasma structure in coexistence with the surrounding main plasma with lower density and higher temperature [8, 9]. Modelling results have shown that radiative condensation is the cause of Marfe formation $[10,11,12]$.

Poloidally localized radiation belts have been observed not only in tokamaks but also in a stellarator and in a heliotron. In the Wendelstein 7-AS stellarator Marfes were found during operation with divertor $[13,14]$ but their properties have not been studied in much detail. A much better studied radiation belt is the so-called Serpent in the heliotron LHD [15] which forms the plasma operational limit in the so-called Serpens mode. The Serpent is also a high-density plasma structure with densities in the range from 2 to $5 * 10^{20} m^{-3}[16,17]$ but has a more complex geometry than the axisymmetric Marfe in a tokamak.

We focus here on measurements in W7-X and a first analysis of the radiation belts in order to understand the physical nature of the phenomenon and the relation to Marfes in tokamaks and Serpents in LHD. In Sec. 2 the plasma diagnostics used to characterize these events are described. In Sec. 3 we describe two different discharge scenarios with

radiation belts in the edge plasma, studied by video camera images in Sec. 4. The density inside the belt was determined by the interferometers, Langmuir probes embedded in one of the limiters and by the filterscope diagnostics. These results are presented in Sec. 5. The toroidal variation of the radiation belt is studied in Sec. 6. Finally, we discuss the physical cause of the radiation belt in Sec. 7 . 


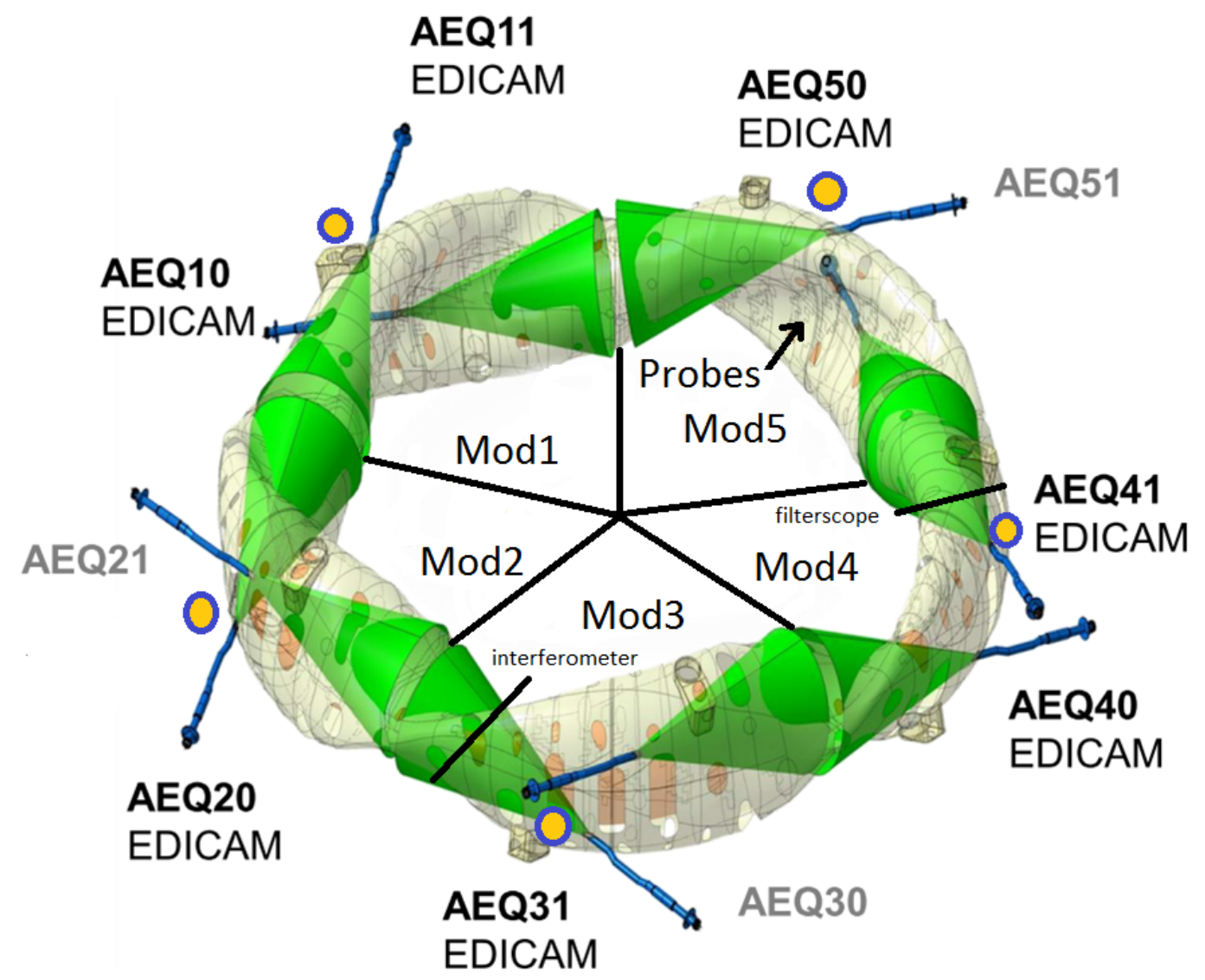

Figure 1. Experimental arrangement of the video cameras (EDICAMs) in the AEQ ports, the interferometer and one filterscope channnel. The position of Langmuir probes in the limiter of module 5 is indicated by the arrow. The five neutral pressure gauges are symbolized by the orange circles. They are approximately at midplane positions. The module designation is abbreviated by Mod1, Mod2, etc.

\section{Experimental setup}

The magnetic field of W7-X exhibits five-fold stellarator symmetry. Each of the five modules consists of two half-modules. Module 1 connects to modules 2 and 5, module 2 to 1 and 3, and so on. Fig. 1 shows the arrangement of the modules from a bird's eye view. The plasma cross section at the module separation points is triangular. The limiters were positioned at the inboard side in the bean-shaped planes, located in the center of each module.

The first experimental campaign on W7-X had a comprehensive set of diagnostics [18]. The main diagnostics for this study were the interferometer, the EDICAM video camera system, the neutral gas manometers, the filterscope (an absolutely calibrated optical line-of-sight spectroscopic diagnostic) and Langmuir probes in one of the limiters.

The single-channel dispersion interferometer was installed for the measurement of the line-integrated density [19]. It measures in module 3 (Port AET31). The laser beam passes through the plasma vessel twice in the radial direction, reflecting after its first 
pass off a corner cube reflector which is located outside the vacuum vessel at the opposite port AEZ31, also in module 3 (see Fig. 1).

The overview video diagnostic system (EDICAM system) was mounted in ten tangential ports of W7-X, giving very good toroidal coverage [20], except in the bean-shaped planes (see Fig. 1). Consequently, the inboard limiters were not directly observable by this diagnostic. The EDICAM camera system was run with $10 \mathrm{~ms}$ temporal resolution during the initial campaign. No interference filters were used; i.e., the EDICAM camera system measured the full visible spectrum.

For the neutral gas density measurements a set of five in-vessel gauges of the ASDEX type were used (for the details, see [21]). The ASDEX Pressure Gauges (APGs) were placed at the outboard mid-plane in all five stellarator modules. The measurement range spans almost five orders of magnitude from $2 \times 10^{-7}$ to $1 \times 10^{-2} \mathrm{mbar}$, with a sampling rate of $2 \mathrm{kHz}[22]$.

The Langmuir probes were mounted in the limiter in module 5 (see Fig. 1). Two arrays with 20 probes in each array were installed in two limiter tiles above and below the equatorial plane. They measured either the floating potential and the ion saturation current at constant bias voltage of $-100 \mathrm{~V}$ or were operated in the sweeping mode with voltages between $-100 \mathrm{~V}$ and $10 \mathrm{~V}$ to measure $n_{e}$ and $T_{e}$, respectively. The time resolution of the probes when operated in swept mode was $2 \mathrm{~ms}$.

The filterscope diagnostic was used for the measurement of the optical emission from the plasma edge $([23])$. Several lines-of-sight were installed at different toroidal positions [24]). These measurements have a high temporal resolution of $10 \mu \mathrm{s}$. For this study, we used only the $\mathrm{H}_{\beta}$ filterscope channel in port AEK41 (in module 4), looking radially through the plasma from the outboard side to the inboard wall roughly in the mid-plane of W7-X (see Fig. 1).

\section{Description of the plasma termination}

When the plasma density was increased significantly by hydrogen gas fueling in OP1.1, the plasmas were often observed to terminate in a radiation collapse, where the minor radial extent of the plasma would shrink over several tens of milliseconds (clearly observable in video camera images). Very similar discharge termination behaviour was also observed during radiation cooling experiments, when a sufficient amount of medium-Z impurities (typically nitrogen) were puffed into the plasma in order to raise the radiated power. We give examples of these two kinds of experiments in the following.

\subsection{Hydrogen gas puffing}

An example of how a plasma termination was triggered by gas puffing is shown in Fig. 2 . The density was raised by hydrogen puffing into the plasma. Three discharges are shown, with the following signals: Total heating power, line-integrated density, neutral pressure 


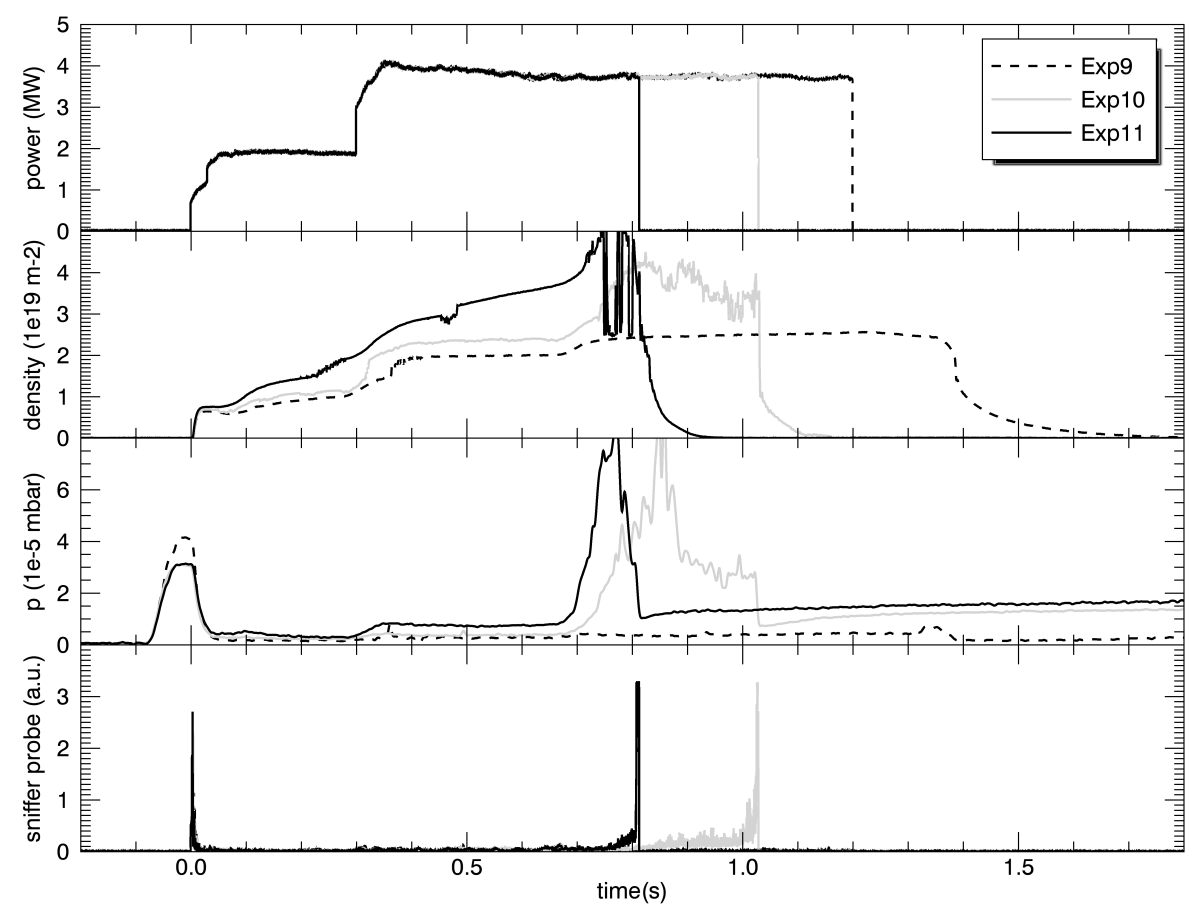

Figure 2. Time traces of 3 consecutive W7-X experiment programs (2016-03-10.9 - 2016-03-10.11) with density ramp-up. The time traces are the total ECRH power, the line-integrated density from the interferometer, the neutral pressure in module 4 and sniffer probe signal. Experiments 10 and 11 were terminated by the sniffer probe before the pre-programmed time of $1.2 \mathrm{~s}$ (switch-off of the ECRH).

measured by the APG in the midplane of module 4 and the signal of the ECR stray radiation detector, which was used as an interlock for the ECR heating. To obtain the line-averaged density, one divides by the interferometer path length of $1.33 \mathrm{~m}$.

Before $\mathrm{t}=0 \mathrm{~s}$ the vacuum vessel was filled with hydrogen up to a pressure of $3.5 \times 10^{-5}$ mbar (prefill). The fill pulse is clearly detected by the APG. At $\mathrm{t}=0$ the plasma is initiated by ECRH. The ECRH power and its pulse length was selected to comply with the administrative limit of $4 \mathrm{MJ}$ per pulse in effect during the last days of operation. From $0.3 \mathrm{~s}$ onward the ECRH power was $4 \mathrm{MW}$. Additional gas fill pulses were pre-programmed during the discharge to raise the density. In experiment 9 we see two plateaus in the electron density corresponding to two additional fill pulses. The neutral pressure shows the plateaus as well. Experiment 9 terminated as pre-programmed.

In the following experiments, 10 and 11, the gas fuel timing was the same but the input was further increased. In both experiments, a large neutral pressure spike was measured in module 4, the line-integrated plasma density increases around the same time, and shortly thereafter, the ECR non-absorbed power shoots up and triggers the interlock, terminating the discharge before its pre-programmed termination. Before the reaction of the sniffer probe the pressure gauge detected a large neutral gas spike which was not 


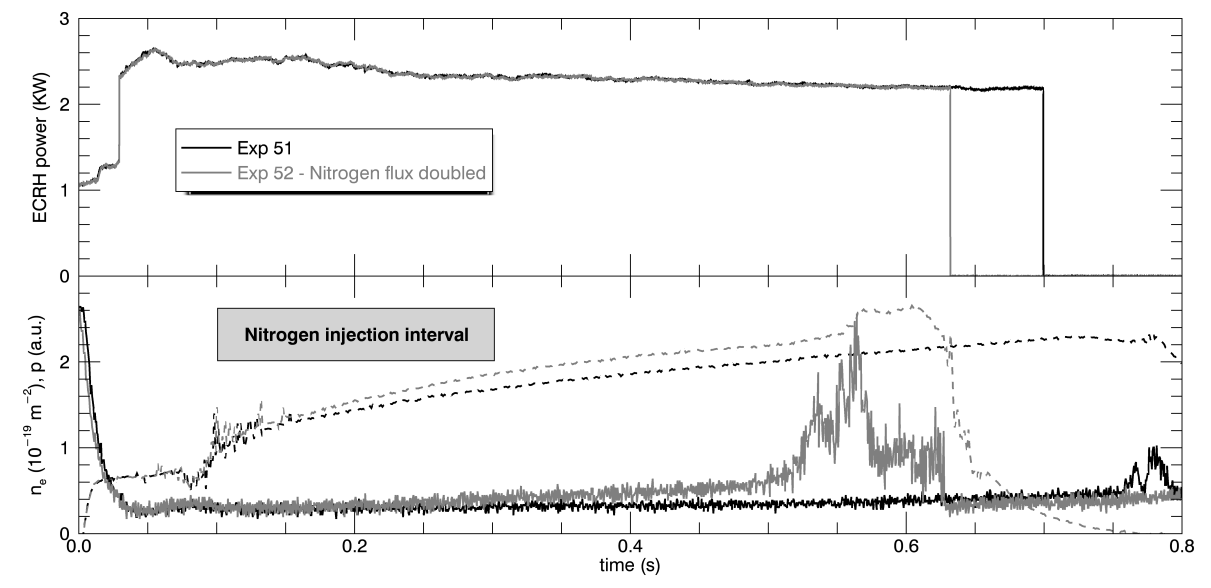

Figure 3. Time traces of two experiments with nitrogen puffing (black - 2016-0309.51 - and gray - 2016-03-09.52): ECRH power, neutral pressure in midplane module 4 (full lines) and the line-integrated electron density (dashed lines). The difference is the amount of injected impurities. In experiment 52 the nitrogen gas flux was doubled compared to experiment 51 . With the doubled amount of nitrogen the plasma survives the impurity injection phase but later suffers a collapse, including a pronounced peak in neutral pressure starting in module 4 .

caused by any injection from a gas valve. This pressure event marked the beginning of the termination of the discharge.

\subsection{Impurity gas puffing}

The same pressure event was also triggered by puffing impurities into the plasma. In these experiments nitrogen was blown into the plasma in small amounts by two injection systems of the thermal helium beam diagnostic. In Fig. 3 two experiments are compared with a different level of nitrogen injection. The time traces show the line-integrated electron density measured by the interferometer and the midplane neutral pressure in module 4. The hydrogen pre-fill pulse was the same in both experiments (as can be seen from the neutral pressure time traces). No other hydrogen gas fuelling was applied.

In both experiments nitrogen was puffed during the same time interval from $100 \mathrm{~ms}$ to $300 \mathrm{~ms}$, but with different flow rates. In experiment 51 the impurity gas was puffed through the injection system in machine module 5 with a flow rate of about $4 \times 10^{19} \mathrm{~s}^{-1}$. In experiment 52 the total flow rate was doubled by adding the same gas flow rate through the injection system in module 3 . In both experiments the density rises continuously after the end of the nitrogen injection, presumably due to wall outgassing, at a rate slightly higher in discharge 52. The preprogrammed ECRH power duration was the same in both discharges: $3 \mathrm{MW}$ for $0.7 \mathrm{~s}$. Experiment 51 was terminated as preprogrammed after $0.7 \mathrm{~s}$ by switching off the ECRH power. In experiment 52 with a slightly higher density we observe again the neutral pressure spike in module 4 with the subsequent plasma termination by the sniffer probe at $0.63 \mathrm{~s}$, i.e. before the 
pre-programmed pulse duration was reached.

\section{Video images}

Fig. 4 shows some selected plasma images of experiment 2016-03-09.52 taken from two different ports AEQ50 (in half-module 50) and AEQ41 (in half-module 41), looking toward each other. From port AEQ50 we observe mainly the half-module 41 and from port AEQ41 mainly the half-module 50. Data are from three different experiment times: $450 \mathrm{~ms}, 550 \mathrm{~ms}$ and $580 \mathrm{~ms}$ (the time resolution of the video diagnostics is $10 \mathrm{~ms}$ ). In the regular plasma phase at $450 \mathrm{~ms}$ the visible plasma emission comes from the plasma edge. As modeling shows [25], the measured emission pattern is that of a poloidally symmetric emission profile. The measured emission is concentrated in one or two regions, which is simply a tangential effect. At $550 \mathrm{~ms}$, in contrast to the regular plasma, we observe a poloidally asymmetric radiation belt. This emission pattern cannot be modeled by a poloidal symmetric emission profile. The radiation belt is similar to a Marfe as observed in tokamaks [8]. This Marfe-like event coincides with the neutral pressure peak in module 4. Note that the radiation distribution in the edge is completely changed. In contrast to the regular plasma at $450 \mathrm{~ms}$ the control coil is visible (see Fig. 4(d)). When the density rises further, the Marfe-like radiation belt disappears and the plasma detaches poloidally (see the images in Fig. 4(e) and Fig. 4(f) at $580 \mathrm{~ms}$ ). Due to the strong poloidally symmetric emission profile the measured emission is concentrated again in two regions (tangential effect). Note that the control coil is not longer visible.

Fig. 5 shows a sequence of 4 subsequent images which show the temporal development of the Marfe-like radiation belt in experiment 2016-03-09.52. The temporal resolution is $10 \mathrm{~ms}$. The emission band is created at the inboard side at $530 \mathrm{~ms}$, around the time of the first abrupt rise in neutral pressure in Module 4. It moves from the inboard to the outboard side within $30 \mathrm{~ms}$ (see images b-d). The rotation direction seen from the AEQ50 camera view is anti-clockwise (in mathematically positive direction).

\section{Plasma edge density}

Let us assume for the moment that the observed radiation belt is a Marfe. Then, during its rotation the plasma edge diagnostics should observe high electron density. We consider here the interferometer and the Langmuir probes.

\subsection{Interferometer}

As already mentioned, the interferometer is installed in module 3 of W7-X. In the image Fig. 5(d) the Marfe-like radiation belt crosses the line-of-sight of the interferometer, projected from module 3 to module 4 , at $560 \mathrm{~ms}$. The crossing point is on the outboard side. Thus, the density bump seen at $560 \mathrm{~ms}$ in the interferometer trace (see Fig. 3) can be explained by the transit of the high-density radiation belt. 

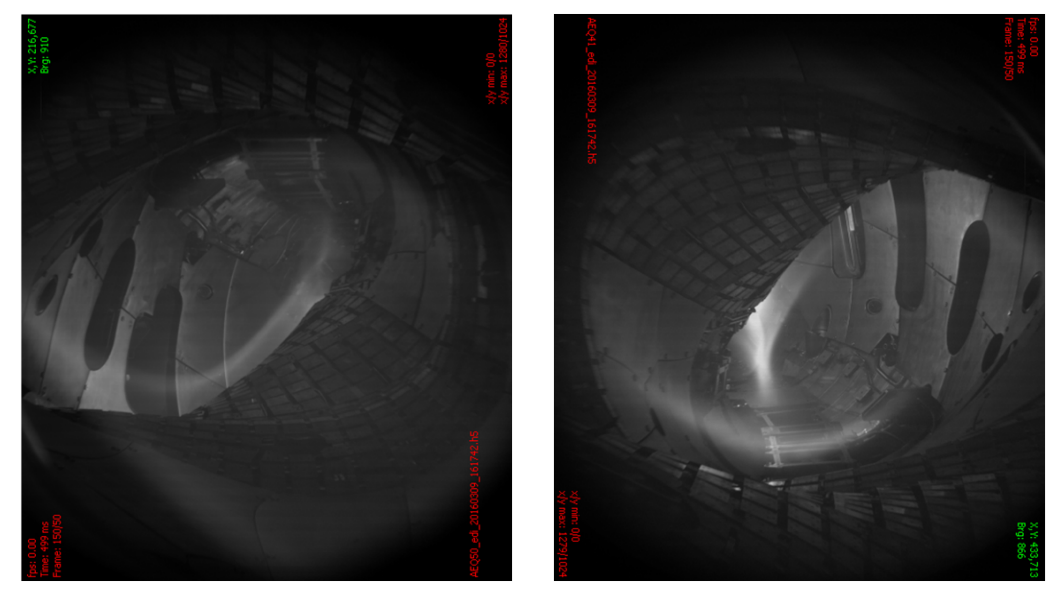

(a) View from AEQ50 at 450 ms: (b) View from AEQ41 at 450 ms: regular plasma regular plasma
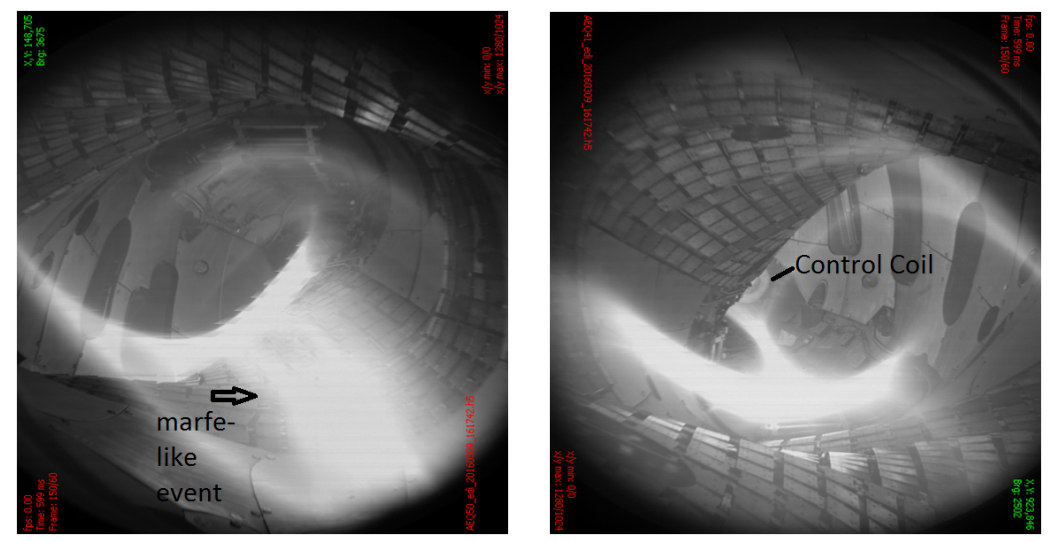

(c) View from AEQ50 at 550 ms: (d) View from AEQ41 at 550 ms: marfe-like radiation belt marfe-like radiation belt
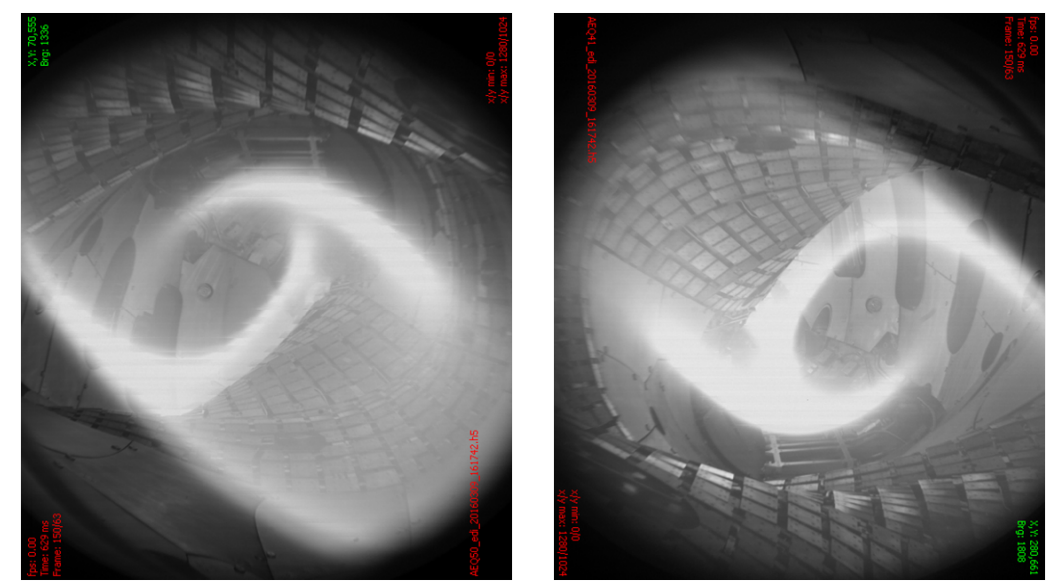

(e) View from AEQ50 at 580 ms: (f) View from AEQ41 at 580 ms: poloidally detached plasma poloidally detached plasma

Figure 4. Selected video images from experiment 2016-03-09.52: regular plasma at $450 \mathrm{~ms}$, Marfe-like radiation belt at $550 \mathrm{~ms}$, and poloidally detached plasma at $580 \mathrm{~ms}$. The limiters are not directly visible in the images. The images cover a fifth of the plasma vessel (half of module 4 and 5). 

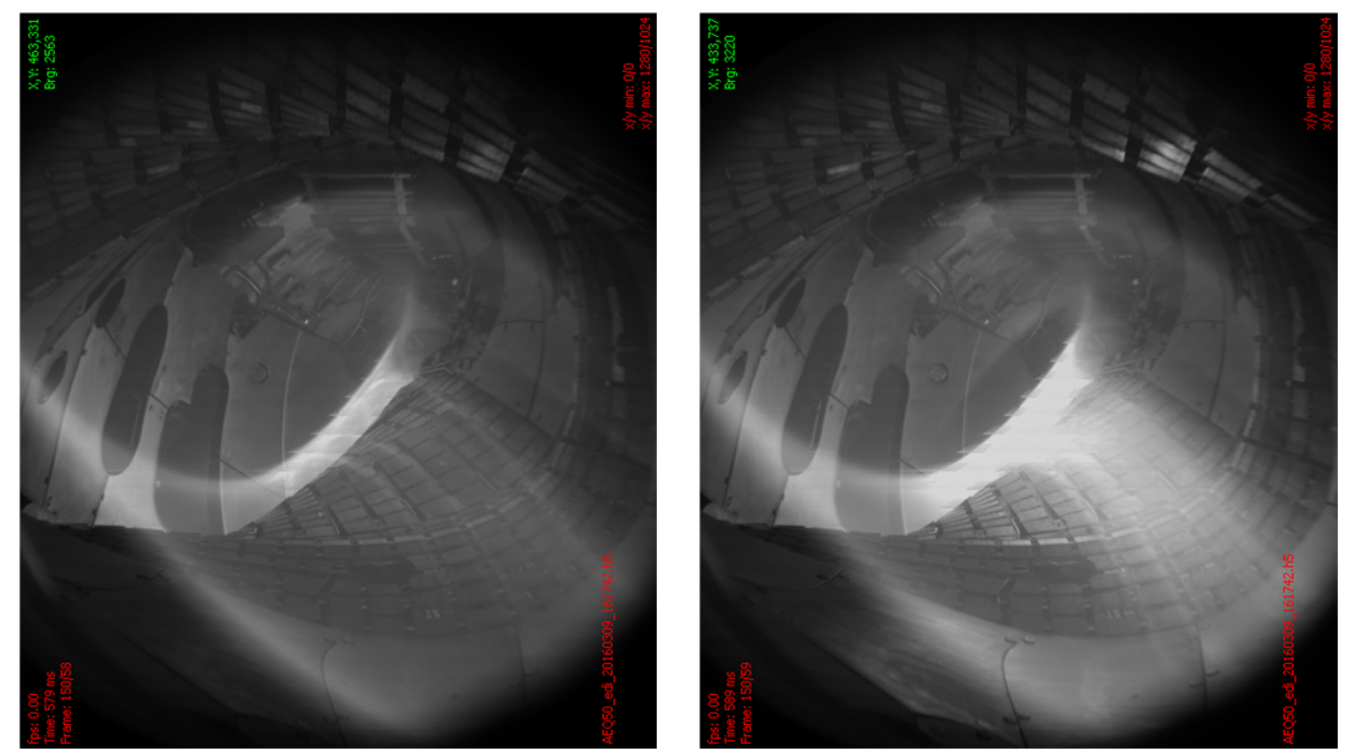

(a) View from AEQ50 at 530 ms: passage of (b) View from AEQ50 at 540 ms: passage of the filterscope channel the filterscope channel
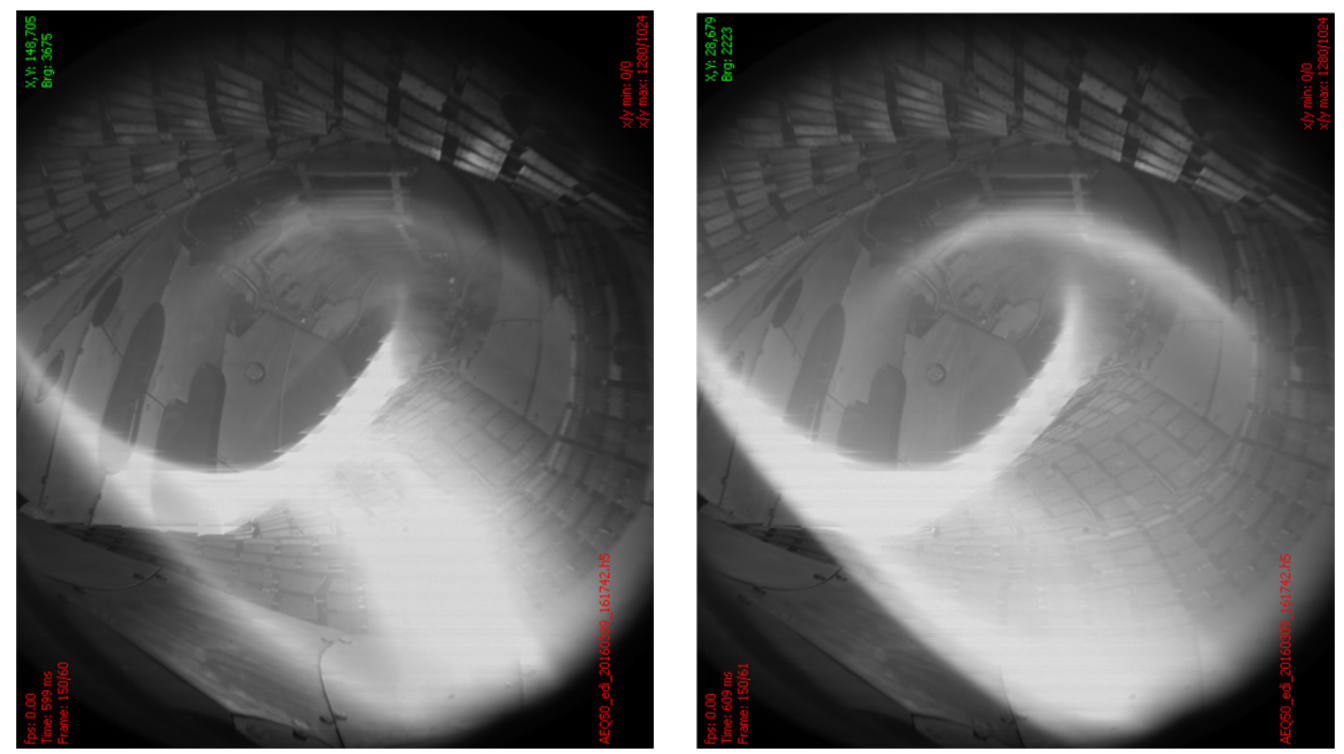

(c) View from AEQ50 at 550 ms: passage of (d) View from AEQ50 at 560 ms: passage of the filterscope channel the interferometer path

Figure 5. Sequence of four video images from experiment 2016-03-09.52 showing the poloidal motion of the Marfe-like radiation belt from the inboard to the outboard side. Due to the rotation it is detected by the interferometer and by the filterscope at different times. 

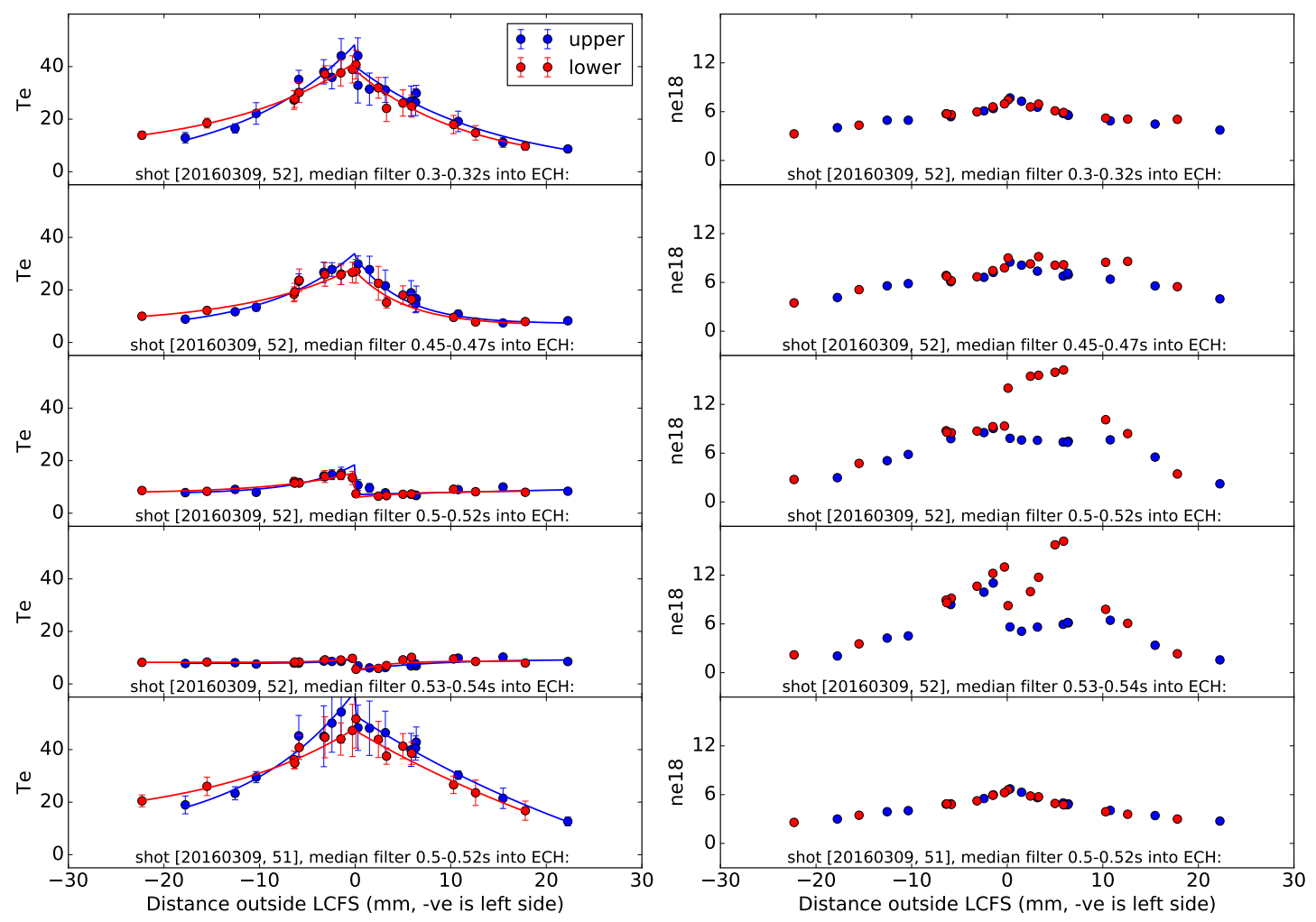

Figure 6. Limiter Langmuir probe results: Some selected temperature (left) and density profiles (right) of experiment 2016-03-09.52. Profiles from the upper limiter are shown in blue, profiles from the lower limiter are shown in red. The first two lines show profiles without the Marfe-like event, the second two lines show profiles with the Marfe-like event. In the last line profiles for experiment 2016-03-09.51 are shown for comparison.

Fig. 3 shows that the line-integrated density rises from 2.3 to $2.6 * 10^{19} \mathrm{~m}^{-2}$ when the radiation belt crosses the interferometer path; i.e., it contributes $0.3 * 10^{19} \mathrm{~m}^{-2}$ to the line-integrated density. With an approximate radial width of the radiation belt of $0.08 \pm 0.02 \mathrm{~m}$ the mean density inside the belt is estimated to about $4 \pm 1 * 10^{19} \mathrm{~m}^{-3}$.

\subsection{Langmuir probe arrays in the limiter}

The Langmuir probes integrated in the limiter tiles also measure edge plasma density. Fig. 6 shows the spatial distribution of the density and electron temperature profiles of experiment 2016-03-09.52 (for four different points in time). In the bottom row, results from experiment 2016-03-09.51 (where no Marfe-like radiation belt was present) are shown for comparison.

The density measured by this array has been corrected for deviations from the nominal probe area, possibly due to erosion or small dimensional changes. For comparison, independent data from the multi-purpose manipulator (MPM [26]) is available for experiment 17 on the same day, but only for distances $35-65 \mathrm{~mm}$ outside the LCFS, requiring extrapolation. Overall, the right side (facing Module 1) of the limiter array 
seems much less affected than the left. From the right side raw data, it would seem that the density data are scattered $+/-20 \%$ about a profile with an e-folding scale length of between 20 and $40 \mathrm{~mm}$. A correction profile is obtained from this data for these scale lengths, adjusted so that when extrapolated, it is consistent with the MPM data. This correction profile makes density profiles over a wide range of shots, powers, and configurations much smoother, and much more left-right symmetric. If the Marfe-like event data are processed by normalisation to the experiment 17 data in this way with each of these assumed profile widths, the Marfe-like event feature time dependence and profiles are essentially unchanged. The main difference is that the $20 \mathrm{~mm}$ profiles are more peaked as expected and have higher peak densities (by about 60\%). Apart from these differences, the time dependence and profile of the Marfe-like event differ only by the approximate size of the point markers.

There are two findings worth noting from the Langmuir probe data.

The first is that the Marfe-like radiation belt is clearly detected on the limiters in the bean-shaped plane - which cannot be observed by the video diagnostic. Thus, the Marfe-like radiation belt is toroidally extended and could be present in all modules. The density profiles from the lower limiter show corresponding density bumps in the profiles from 0.5-0.52 and 0.53-0.54 s while the profiles from the upper limiter are unchanged during these time intervals. The Marfe-like radiation belt touches the limiter only in the lower region below midplane. We assume that this is the case for all five limiters because of the similarity of the video images in all modules. It is not clear whether the Marfe is principally confined to the lower region in W7-X or can freely rotate. In the latter case we would observe it also in the upper part of the torus. A comparison of the two density profiles with the Marfe feature from the lower limiter shows that Marfe-like radiation belt moves downward. This is illustrated in Fig. 7 where the corresponding probes are marked which detect the enhanced density in the interval $0.53-0.54 \mathrm{~s}$. The maximum density is detected by the probes 5 and 6 . It is about $1.5 \times 10^{19} \mathrm{~m}^{-3}$ which is still relatively low compared to the estimation of the density from the bump in the interferometer trace.

The second thing worth noting is the direct measurement of the temperature at and just barely outside the separatrix, which is measured by the Langmuir probes near the limiter centre. In experiment 51 the maximum temperature is about $50 \mathrm{eV}$. In experiment 52 the temperature is about $30 \mathrm{eV}$ prior the Marfe-like radiation belt (see the temperature profile from $0.45-0.47 \mathrm{~s}$ ). This supports our conclusion that it is a radiative condensation phenomenon which appears below a certain edge temperature, as we will discuss in Section 7. By puffing nitrogen impurities into the plasma edge with a sufficient puffing rate, it is cooled to below the threshold.

\subsection{Filterscope results}

In Fig. 8 the neutral pressure in module 4 is compared to the filterscope diagnostic from port AEK41. We show the filterscope signal with an $\mathrm{H}_{\beta}$ interference filter from 


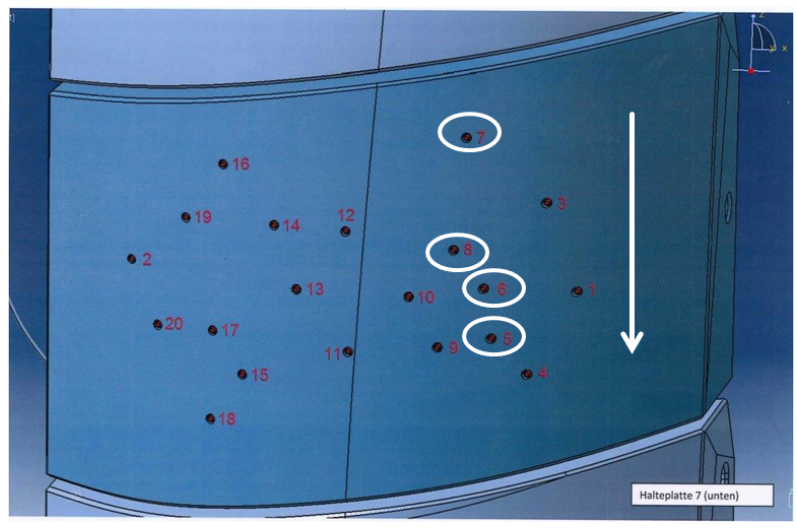

Figure 7. The positions of the four Langmuir probes in the lower limiter which detect the Marfe-like radiation belt between 0.53 and $0.54 \mathrm{~s}$. The maximum density is measured by the probes 5 and 6 (compared to the probes $5,6,7$ and 8 in the interval before) which suggests a downward movement in the bean-shaped plane of module 5 as indicated by the arrow.

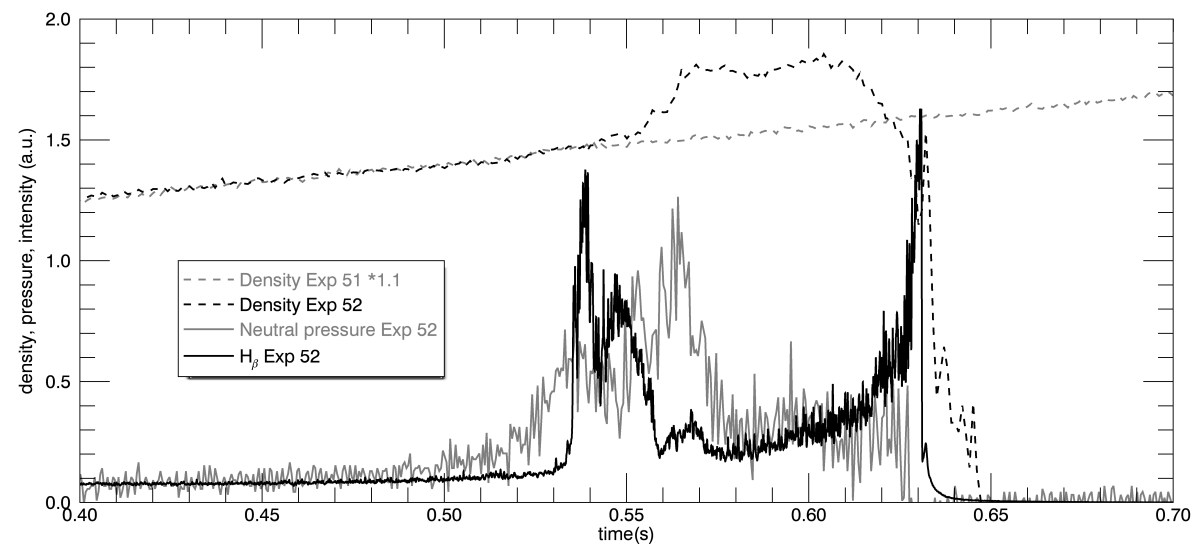

Figure 8. Time trace of one filterscope channel from port AEK41 with $\mathrm{H}_{\beta}$ filter. The neutral pressure (module 4) and the interferometer time traces are also shown for comparison. The Marfe-like radiation belt exists from 530-570 ms. When it passes filterscope channel (from $530-560 \mathrm{~ms}$ ) the $\mathrm{H}_{\beta}$ signal strongly rises.

experiment 2016-03-09.52. The $\mathrm{H}_{\beta}$ signal is very large when the radiation belt crosses the filterscope channel at 540 and $550 \mathrm{~ms}$. At $560 \mathrm{~ms}$, however, the radiation belt is out of the filterscope line-of-sight and the signal is correspondingly smaller (compare the images in Fig. 5).

There is another typical observation of an $\mathrm{H}_{\beta}$ rise at the end of the experiment when the heating is switched-off and the charged particles are still confined in the magnetic field. In this phase of the experiment the density remains high [19] but the particles cool down and recombine. The increased in $\mathrm{H}_{\beta}$ is the consequence of the volume recombination. We think that the strong rise of $\mathrm{H}_{\beta}$ in the Marfe-like radiation belt has the same cause. 

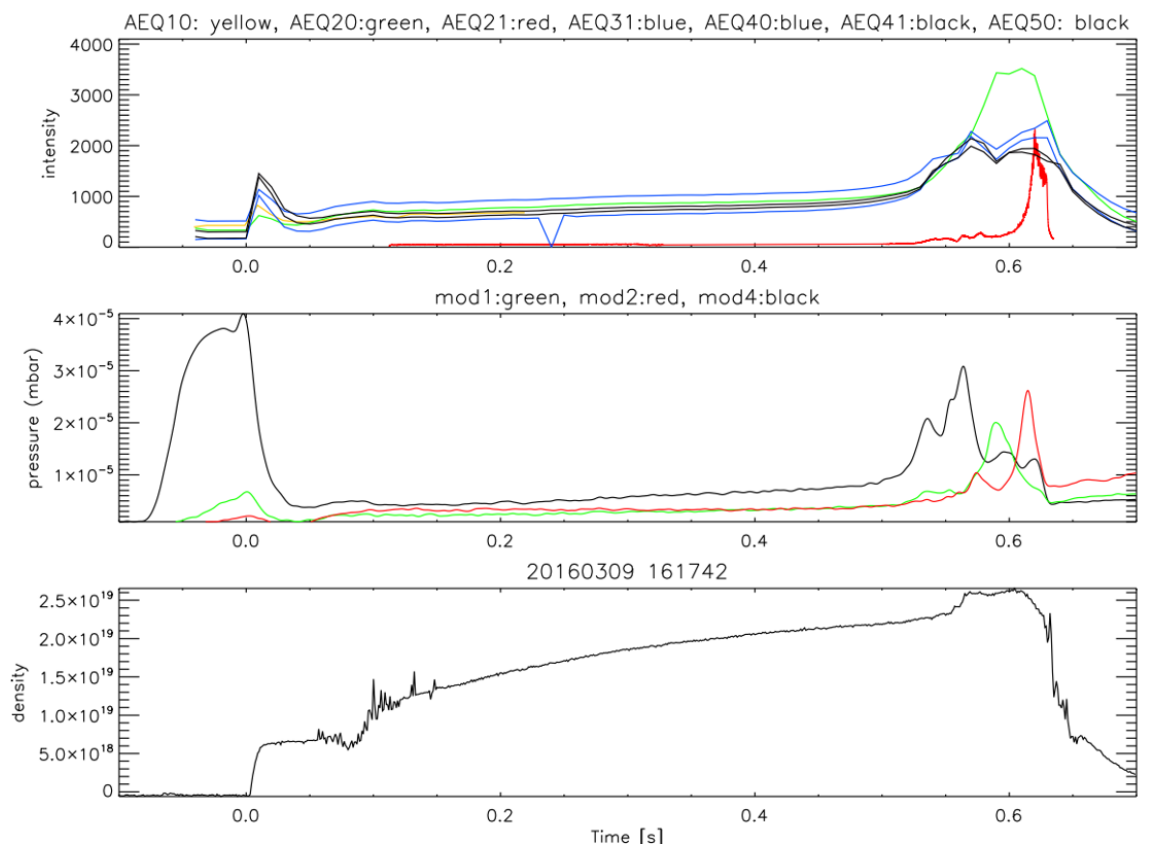

Figure 9. Temporal behaviour of the radiation belt $(\mathrm{t}=550-630 \mathrm{~ms})$. Data is from experiment 2016-03-09.52. The total emission as measured by seven video cameras (on top) is roughly correlated with the neutral pressure from 3 modules (in the middle). Data shows a toroidal variation of neutral pressure and visible emission inside the Marfe-like radiation belt. The line-integrated density is plotted for comparison (at bottom).

\section{Toroidal variation of the Marfe-like radiation belt}

The emission pattern of the radiation belt seen from equivalent positions in the other stellarator modules is very similar. This implies that it has the five-fold sysmmetry of the magnetic field. The intensity, however, exhibits a spatio-temporal variation.

Fig. 9 shows the intensity of the video images and the neutral pressure as a function of time. Note that the optical emission has no spectral resolution. The whole visible emission was summed upon the CCD sensor. The neutral pressure spike appears first in module 4 and moves in positive, anti-clockwise toroidal direction over module 1 towards module 2 (seen from the bird's eye view). As Fig. 9 shows, there is a rough correlation between the total optical emission and the neutral pressure. There is not only a poloidal rotation but also a toroidal rotation of a high-emission, high-neutral-pressure feature inside the radiation belt. A toroidal movement of a high density region with volume recombination region would explain both features.

\section{Discussion}

At the beginning of the discussion we would like to emphasize that the experimentally found properties of the radiation belt in W7-X are very similar to a Marfe: 
- Both exhibit the device symmetry, namely axisymmetry in a tokamak and the five-fold stellarator symmetry in W7-X.

- The radiation belts have a higher electron density compared to the the surrounding plasma.

- Volume recombination is detected in the radiation belts, indicating that the temperature inside must be very low.

We have not directly proven the latter item, which would require a simultaneous measurement of density and temperature in the center of the belt. However, we have detected the pressure spikes and high $\mathrm{H}_{\beta}$ emission, both of which are very hard to understand without te assumption of volume recombination. Because of these coincidences, we conclude that the radiation belt in W7-X is a Marfe with some special properties. These are the poloidal rotation and the toroidal modulation of the belt; i.e., of the neutral pressure and the total emission.

A Marfe is the result of a radiative condensation process which is possible at a certain radiation level. We have found an approximate mean density in the radiation belt of $4 * 10^{19} \mathrm{~m}^{-3}$, which is much lower than the estimated density inside the Serpent of LHD $\left(2 . .5 * 10^{20} \mathrm{~m}^{-3}\right)$. An improved measurement of the plasma parameters inside the Marfe in W7-X remains a topic of future work, but nonetheless this value indicates the condensation.

This picture is supported by model calculations for a cylindrical equilibrium which remained thermically stable up to a critical radiation rate where the power input into the plasma edge equals the totally radiated power [27]. In the model, radiative condensation already started at $75 \%$ of the loss power; i.e., well below the critical radiation fraction of $100 \%$. This results explains why we observe the Marfe-like radiation belt before the onset of the full poloidal detachment with shrinking plasma radius (see Fig. 5).

The critical power threshold is mainly a temperature threshold. The total radiation $P_{t o t}$ per unit volume is given by $P_{t o t}=L_{Z}(T) * n_{e} * n_{Z}$, where $L_{Z}(T)$ is the radiative power function, $n_{e}$ is the electron density, and $n_{i}$ is the ion density. In the absence of strong nitrogen puffing, the dominant edge impurity was carbon in W7-X limiter operation, owing to the fact that the limiters were made of fine-grain graphite. The radiation level depends linearly on the impurity density but is a nonlinear function of electron temperature - see Fig. 10. For W7-X at the edge, where the Langmuir probes indicate that $n_{e} \approx 5 \times 10^{18} \mathrm{~m}^{-3}$, the $n_{e} * \tau_{i}=10^{18} \mathrm{~m}^{-3} \mathrm{~s}$ curve should be the most relevant one, since we can safely assume $\tau_{I} \geq 200 \mathrm{~ms}$. It has an unstable slope between about $8 \mathrm{eV}$ and $T_{\min }=40 \mathrm{eV}$ where it varies by three orders of magnitude. By unstable it is meant that a lower $T_{e}$ will lead to stronger radiative losses, which will lower the temperature further, thus making the plasma temperature collapse into the $T_{e} \leq 1 \mathrm{eV}$ range. The radiative power function for nitrogen, used in the impurity gas puff experiments (Sec. 3.2 ), is similar to that of carbon and has also a minimum of the radiative power function at $40 \mathrm{eV}$. Our Langmuir probe measurements of electron temperature support the notion that radiative condensation is initiated at a critical temperature. In the stable discharge, 


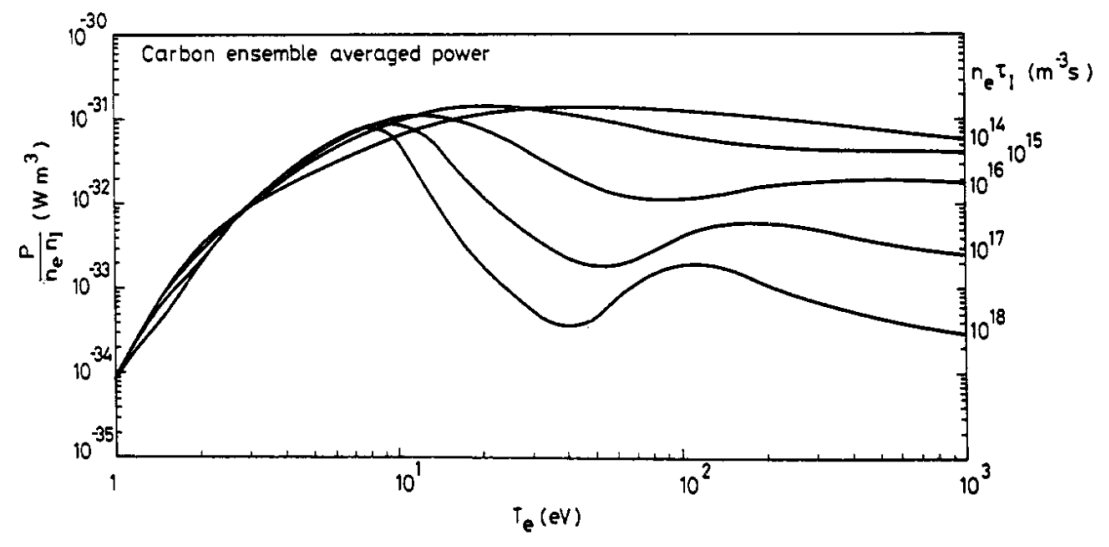

Figure 10. The radiative power function of carbon as a function of the electron temperature [28] for different values of $n_{e} * \tau_{i}$, where $\tau_{i}$ is the impurity ion confinement time. For larger values of $n_{e} * \tau_{i}$, the function approaches that predicted by coronal equilibrium. Approaching this limit, a local minimum appears. At $n_{e} * \tau_{i}=10^{18} \mathrm{~m}^{-3} \mathrm{~s}$, realistic for $\mathrm{W} 7-\mathrm{X}$ edge conditions, the minimum is around $\mathrm{Tmin} \approx 40 \mathrm{eV}$.

the edge electron temperature remained around $50 \mathrm{eV}$ whereas it was about $30 \mathrm{eV}$ in the discharge that showed the Marfe.

A special feature is the observed poloidal rotation of the Marfe to the outboard side. Marfes in circular tokamaks can also move upwards or downwards when they reside in front of the inboard wall $[29,30]$. They were, however, never observed on the outboard side. In LHD the Serpent completed many full rotations over several seconds [15]. Due to the rotation several plasma edge diagnostic signals exhibited strong fluctuations. The poloidal rotation frequency could be determined to be about several tens of ms depending on the heating power. In stellarators and heliotrons Marfes are observed on the outboard side or can poloidally rotate.

As already mentioned, it is very likely that the observed pressured spikes are due to volume recombination, but a number of open questions remain for a comprehensive understanding of this phenomenon. One is why the first spike always appeared in module 4.

During operation, it was observed that these pressure spikes and subsequent early terminations of the plasma would appear progressively earlier in the discharges as the run day went on. Wall-cleaning with helium discharges would often recover good performance, and glow-discharge cleaning would always do so. Thus, there is evidence that these events were also related to excessive outgassing from the walls, possibly even self-consistently triggered by high-density low-temperature plasma edge conditions, because these imply a larger fraction of the power losses going to components other than the limiters - be it through charge-exhange neutrals, radiation photons, or even a widened scrape-off layer.

One hypothesis is that an initial, localized, and abrupt influx of neutrals starting around $\mathrm{t}=0.52 \mathrm{~s}$ causes a local plasma density increase, a resulting local temperature decrease, 
and the Marfe is triggered into existence. It then becomes itself a source of neutrals, through volume recombination. Although some molten plastic was found in a few places in the device, which could act as such localized sources if heated above their evaporation temperatures, none were found in module 4, so the nature and existence of such a source remains unclear. Another hypothesis is that the Marfe is triggered, not by an abrupt influx of neutrals, but by a gradually dropping electron temperature, and that the Marfe is the abrupt and local source of neutrals detected starting at $t=0.52 \mathrm{~s}$. This second interpretation conflicts, however, with the observation that the pressure seems to rise first followed by the the sudden increase of $H_{\beta}$ (see Fig. 8).

One conclusion regarding sources of neutrals from the outside can be drawn: There must be some additional - and significant - external source of particles involved in the dynamics from 0.52 to 0.6 seconds in experiment 52 . This follows from the fact that the recombination process is simultaneously a source of neutral particles, and an equivalent sink for the plasma particles. Since both electron density and neutral pressure go up during this time period, an extra external particle source must be present.

\section{Summary}

A number of discharges in the W7-X operational phase 1.1 with inboard limiters were terminated by radiative condensation that featured a Marfe-like radiation belt at the edge and one or several neutral pressure spikes due to volume recombination. The higher density inside this event was detected by the interferometer and by Langmuir probes. Marfe-like radiation belts in W7-X are non-axisymmetric and have the five-fold stellarator symmetry. They appear to be triggered at an edge electron temperature of $30 \mathrm{eV}$, consistent with the radiation characteristics of carbon and nitrogen near coronal equilibrium. The drop in edge temperature was, in the operational phase 1.1, caused by an increased plasma density or increased impurity radiation, and was associated with an external neutral particle source whose exact nature remains unclear. In the operational

phase 1.2 with increased plasma density, but also strongly increased heating power, and an island divertor, with a better core-edge separation and more efficient pumping, as well as more elaborate wall-conditioning, Marfe-like radiation belts were also observed, especially in the high-iota configuration. It remains to be seen if they can be avoided in certain magnetic configurations.

\section{Acknowledgement}

This work has been carried out within the framework of the EUROfusion Consortium and has received funding from the Euratom research and training programme 20142018 under grant agreement No 633053. The views and opinions expressed herein do not necessarily reflect those of the European Commission. 


\section{References}

[1] Grieger G, Lotz W, Merkel P, Nührenberg J, Sapper J, Strumberger E, Wobig H, Burhenn R, Erckmann V, Gasparino U, Giannone L, Hartfuss H J, Jaenicke R, Kühner G, Ringler H, Weller A, Wagner F, the W7-X Team and the W7-AS Team 1992 Physics of Fluids B: Plasma Physics 4 2081-2091 (Preprint http://dx.doi.org/10.1063/1.860481)

[2] Pedersen T S, Dinklage A, Turkin Y, Wolf R, Bozhenkov S, Geiger J, Fuchert G, Bosch H S, Rahbarnia K, Thomsen H, Neuner U, Klinger T, Langenberg A, Mora H T, Kornejew P, Knauer J, Hirsch M, Pablant N and the W7-X Team 2017 Physics of Plasmas 24055503 (Preprint https://doi.org/10.1063/1.4983629)

[3] Lipschultz B 1984 Nucl. Fusion 24 977-988 ISSN 0029-5515

[4] Murari A, Camplani M, Cannas B, Mazon D, Delaunay F, Usai P and Delmond J F 2010 IEEE Transactions on Plasma Science 38 3409-3418 ISSN 0093-3813

[5] Mertens V, Junker W, Laux M, Schittenhelm M, Büchl K, Mast F, Carlson A, Field A, Fuchs C, Gehre O, Gruber O, Hermann A, Haas G, Kallenbach A, Kaufmann M, Koppendörfer W, Lackner K, Lieder G, Pitcher S, Neuhauser J, Ryter F, Salzmann H, Sandmann W, Steuer K H, Weinlich M, Wenzel U, Zohm H and Team A U 1994 Plasma Physics and Controlled Fusion 36 1307 URL http://stacks.iop.org/0741-3335/36/i=8/a=006

[6] Frigione D, Pieroni L, Zanza V, Apruzzese G, Alladio F, Apicella M, Bartiromo R, Borra M, Bracco G, Buceti G, Buratti P, Centioli C, Ciotti M, Cocilovo V, Condrea I, Crisanti F, de Angelis R, Esposito B, Frattolillo A, Gatti G, Giovannozzi E, Granucci G, Grolli M, Imparato A, Kroegler H, Leigheb M, Lovisetto L, Maddaluno G, Mazzitelli G, Micozzi P, Migliori S, Moleti A, Orsitto F, Pacella D, Panaccione L, Panella M, Pericoli-Ridolfini V, Podda S, Righetti G, Sternini E, Tuccillo A, Tudisco O, Valente F, Vitale V and Zerbini M 1996 Nuclear Fusion 361489 URL http://stacks.iop.org/0029-5515/36/i=11/a=I04

[7] Samm U, Brix M, Durodie F, Lehnen M, Pospieszczyk A, Rapp J, Sergienko G, Schweer B, Tokar $\mathrm{M}$ and Unterberg B $1999 \mathrm{~J}$. Nucl. Mat. 266-269 666

[8] Lipschultz B, Terry J, Boswell A and Hubbard B 1998 Phys. Rev. Lett. 81 1007-1010

[9] Sergienko G, Pospieszczyk A, Lehnen M, Brix M, Rapp J, Schweer B and Greenland P 2001 J. Nucl. Mat. 290-293 720-724

[10] Drake J 1987 Phys. Fluids 30 2429-2433

[11] Kastelewicz H, Schneider R and Neuhauser J 1995 Plasma Phys. Control. Fusion 37 $723-739$

[12] Kotov V and Reiter D 2012 Plasma Phys. Control. Fusion 54 1-4

[13] Wenzel U, McCormick K, Hildebrandt D, Klose S, Naujoks D and Thomsen H 2002 Plasma Phys. Control. Fusion 44 L57-L62

[14] Thomsen H, König R, Feng Y, Grigull P, Klinger T, McCormick K, Ramasubramanian N and Wenzel U 2004 Nuclear Fusion 44 820-826

[15] Masuzaki S, Sakamoto R, Miyazawa Y, Tamura N, Peterson B and the LHD Experimental Group 2005 J. Plasma Fusion Res. 81 649-650

[16] Goto M and Morita S 2008 Plasma and Fusion Research 3 S1042-1-S1042-5

[17] Sakamoto, R and Masuzaki, S and Miyazawa, J and LHD Experimental Group 2006 Plasma and Fusion Research 1 019-1-019-2

[18] Krychowiak M, Adnan A, Alonso A, Andreeva T, Baldzuhn J, Barbui T, Beurskens M, Biel W, Biedermann C, Blackwell B D, Bosch H S, Bozhenkov S, Brakel R, Bräuer T, de Carvalho B B, Burhenn R, Buttenschön B, Cappa A, Cseh G, Czarnecka A, Dinklage A, Drews P, Dzikowicka A, Effenberg F, Endler M, Erckmann V, Estrada T, Ford O, Fornal T, Frerichs H, Fuchert G, Geiger J, Grulke O, Harris J H, Hartfü̈ H J, Hartmann D, Hathiramani D, Hirsch M, Höfel U, Jablonski S, Jakubowski M W, Kaczmarczyk J, Klinger T, Klose S, Knauer J, Kocsis G, König R, Kornejew P, Krämer-Flecken A, Krawczyk N, Kremeyer T, Ksiazek I, Kubkowska M, Langenberg A, Laqua H P, Laux M, Lazerson S, Liang Y, Liu S C, Lorenz A, Marchuk A O, Marsen S, Moncada V, Naujoks D, Neilson H, Neubauer O, Neuner U, Niemann H, 
Oosterbeek J W, Otte M, Pablant N, Pasch E, Pedersen T S, Pisano F, Rahbarnia K, Ryc L, Schmitz O, Schmuck S, Schneider W, Schröder T, Schuhmacher H, Schweer B, Standley B, Stange T, Stephey L, Svensson J, Szabolics T, Szepesi T, Thomsen H, Travere J M, Mora H T, Tsuchiya H, Weir G M, Wenzel U, Werner A, Wiegel B, Windisch T, Wolf R, Wurden G A, Zhang D, Zimbal A and Zoletnik S 2016 Review of Scientific Instruments 87 11D304 (Preprint http://aip.scitation.org/doi/pdf/10.1063/1.4964376)

[19] Knauer J, Kornejew P, Trimio Mora H, Hirsch M, Werner A, Wolf R and the W7-X Team 2016 A new dispersion interferometer for the stellarator Wendelstein 7-X Europhysics Conference Abstracts, Proc. of the 43rd EPS Conference on Controlled Fusion and Plasma Physics, Leuven, Belgium p4.017 URL ocs.ciemat.es/EPS2016PAP/pdf/P4.017.pdf

[20] Kocsis G, Baross T, Biedermann C, Bodnr G, Cseh G, Ilkei T, König R, Otte M, Szabolics T, Szepesi T and Zoletnik S 2015 Fusion Engineering and Design 96-97 808-811

[21] Haas G and Bosch H S 1998 Vacuum 51 39-46

[22] Wenzel U, Kremeyer T, Schlisio G, Marquardt M, Pedersen T, Schmitz O, Mackie B, MaisanoBrown J and the W7-X team 2017 Journal of Instrumentation $12 \mathrm{C} 09008$

[23] Brooks N 2008 Review of Scientific Instruments 79 10F330

[24] Stephey L, Wurden G, Schmitz O, Frerichs H, Effenberg F, Biedermann C, Harris J, König R, Kornejew P, Krychowiak M, Unterberg E and the W7-X Team 2016 Review of Scientific Instruments 87 11D606

[25] Szepesi T, Biedermann C, Blackwell B, Bozhenkov S, Cseh G, Effenberg F, Fuchert G, Höfel U, Kocsis G, König R, Pedersen T, Drews P, Wenzel U, Zoletnik S and the W7-X Team 2017 In 44th EPS Conference on Plasma Physics. Geneva: European Physical Society. (Preprint http://hdl.handle.net/21.11116/0000-0000-B304-1)

[26] Liang Y, Neubauer O, Konig R, Krychowiak M, Schweer B, Denner P, Rack M, Reiter D, Feng Y, Krämer-Flecken A, Drews P, Hasenbeck F, Liu S, Gao Y, Wang E, Wei Y, Dostal M, Li L, Wang N and Linsmeier C 2017 Nuclear Fusion 57066049

[27] Drake J 1987 The Physics of Fluids 30 2429-2433

[28] Carolan A and Piotrowicz V 1983 Plasma Physics 25 1065-1086

[29] Chankin A 2004 Phys. Plasm. 11 1484-1492

[30] Mazzotta C, Spizzo G, Pucella G, Giovannozzi E, Tudisco O, Apruzzese G, Bin W and Esposito B 2017 Nuclear Materials and Energy 12808 - 812 ISSN 2352-1791 proceedings of the 22nd International Conference on Plasma Surface Interactions 2016, 22nd PSI URL http://www.sciencedirect.com/science/article/pii/S235217911630268X 